\title{
Source Detection Performance Prediction for a CdZnTe Array
}

\author{
Daniel J. Lingenfelter, Student Member, IEEE, Jeffrey A. Fessler, Fellow, IEEE, Clayton D. Scott, Member, IEEE, \\ and Zhong He, Senior Member, IEEE
}

\begin{abstract}
The complex system response of 3D position-sensitive gamma-ray detectors complicates modeling the recorded measurements and makes exact expressions for detection performance intractable. This makes source detection performance difficult and expensive to compute. Asymptotic analysis has the potential to simplify detection performance prediction with complex systems and has previously been applied to detection performance prediction with simulated gamma-ray detectors. In this work, we use asymptotic performance prediction methods to predict points on the receiver operating characteristic (ROC) curve for the illustrative task of detecting a ${ }^{137} \mathrm{Cs}$ source in background with an 18-detector CdZnTe array. We assume that the source position, source energy, background spectrum, and background spatial distribution are known. Although these assumptions are not always valid in practice, the efficiency of the prediction method makes it attractive for detection system design problems. Our results show that the asymptotic performance prediction method accurately predicts the empirically observed performance with real data recorded with a real system. Our results also characterize the performance of the detector array for the task of source detection. The accuracy and computational efficiency of the asymptotic detection performance prediction method make it a viable alternative to empirical performance evaluation.
\end{abstract}

Index Terms-Asymptotics, Compton imaging, performance prediction, receiver operating characteristic, source detection.

\section{INTRODUCTION}

G AMMA-RAY source detection problems arise in security screening, nuclear nonproliferation, and medical diagnostics. Simple systems for radioactive source detection look for an increase in the rate of received photons due to a radiation source. More complex measurement systems use spatial and spectral information to more accurately detect radiation sources, but these systems often have a complicated system response,

Manuscript received December 13, 2011; revised August 05, 2012; accepted December 06, 2012. Date of publication January 30, 2013; date of current version February 06, 2013. This work was supported by the U.S. Department of Homeland Security-DNDO under Grant Award Number 2008-DN-077-ARI007-03, the MIT Lincoln Laboratory Fellowship, and the Innovative Signal Analysis Fellowship.

D. J. Lingenfelter, J. A. Fessler, and C. D. Scott are with the Department of Electrical Engineering and Computer Science, University of Michigan, Ann Arbor, MI 48109 USA (e-mail: danling@umich.edu; fessler@umich.edu; clayscot@umich.edu).

Z. He is with the Department of Nuclear Engineering and Radiological Sciences, University of Michigan, Ann Arbor, MI 48109 USA (e-mail: hezhong@umich.edu).

Color versions of one or more of the figures in this paper are available online at http://ieeexplore.ieee.org.

Digital Object Identifier 10.1109/TNS.2012.2236358 making it difficult to compute detection performance analytically. The 3D position-sensitive detector and its system response in [1] illustrate this point.

In this work, we quantify detection performance in terms of points on the receiver operating characteristic (ROC) curve, which is the probability of detection as a function of the probability of false alarm [2]. Previous work that characterized the detection performance of gamma-ray detectors relied on empirical ROC calculation, e.g., [3], [4]. Empirical ROC calculation is performed by simulating multiple scans with a detector, and using the statistics of those scans to compute the probabilities of false alarm and detection. Empirical ROC calculation is computationally expensive and provides only limited intuition about how detector or environment parameters affect detection performance. However, empirical ROC computation may be preferable when recorded data is readily available or highly accurate predictions are necessary.

Asymptotic ROC prediction is a computationally efficient alternative to empirical ROC computation for likelihood-based tests, or tests that are functions of estimates obtained by maximizing a modeled likelihood. We developed asymptotic approximations for the distributions of likelihood-based estimates in [5], and used these approximations to predict detection performance in the presence of model mismatch. It was shown in [5] that the asymptotic performance prediction method yields more accurate predictions in terms of mean-square error than empirical methods, especially when few measurements are available. It was also shown in [5] that ROC predictions based on asymptotics achieve the same accuracy, in terms of mean-squared error of the area under the ROC, with fewer recorded or simulated events. This reduces the computational cost of producing the ROC curve because system response calculations can be expensive.

The simulation results of [5] do not demonstrate that the proposed method can accurately predict the performance of real detectors. In this work, we show that the performance prediction method that accounts for model mismatch developed in [5] can accurately predict source detection performance with a real Compton imaging system. To our knowledge, this work is the first to successfully apply an asymptotic performance prediction method to characterize the performance of the source detection task with a real gamma-ray imaging system.

In addition to demonstrating the practical utility of the asymptotic performance predictions of [5], this work serves as an example application of the asymptotic performance prediction method using real data. We use the asymptotic method to predict the probability of detection as a function of scan time 
with a fixed false-alarm rate for various source-to-background ratios. These examples demonstrate how the asymptotic performance prediction method can be applied to evaluate the performance of real detectors in the field.

Demonstrating the accuracy of the asymptotic performance prediction method with real data is significant because there is more model mismatch than in the simulated case. For example, Doppler broadening ([6], p. 311) is not simulated in [5]. Room-temperature pixellated semiconductor detectors, including the detectors used in this work [7], have an area near the anode where interacting photons are not detected. To simplify computation, the model used in this work, based on [1], does not account for this non-ideal detector behavior. Furthermore, crystal defects can cause errors in the measured interaction positions that are not accounted for by the model. Our results show that the asymptotic prediction method is reasonably accurate in the scenarios considered despite the system response approximation and failure to account for all non-ideal detector behavior.

The contributions of this work are: (i) to show that the asymptotic performance prediction method developed in [5] gives reasonable predictions with a real system, (ii) to illustrate practical uses of this method, and (iii) to provide representative detection performance figures for a real $\mathrm{CdZnTe}$ gamma-ray imaging system. This paper is organized as follows: Section II describes the experimental setup, Section III shows predicted and empirical performance of the detector for various tasks, and Section IV gives our conclusions and plans for future work.

\section{METHODS}

We recorded gamma-ray interaction data with a Compton imaging system consisting of an 18-detector CdZnTe array similar to the system described in [8]. We obtained list-mode measurements of the natural background in a room with concrete walls, and measurements in the same position with a $1-\mu \mathrm{Ci} \mathrm{Cs}$ source located 1.83 meters from the front of the detector. We used the events obtained from these measurements to evaluate the source detection performance of the system.

\section{A. Measurement Model}

There are many aspects of the gamma-ray source detection problem that one can model. The system model and sensitivity are necessary for the likelihood-based detection methods used in this work. We also model the background spatial and energy distributions because this improves detection performance when the modeling is reasonably accurate.

1) Model Parameters: There are several parameters that characterize the gamma-ray source detection problem. We characterize the source by its intensity $\alpha$ with units of gamma-rays emitted per unit time, position $\phi$, which lies in the space $\Phi$ of possible source positions, and energy $e$, which lies in the space of possible energies $\mathcal{E}$. In the 3D far-field with a known source energy, the set $\Phi$ could be $[0,2 \pi] \times[0, \pi]$, representing all possible azimuth and polar angles on a sphere. The set of possible source positions $\Phi$ is independent of the detector. We parameterize the background intensity by the background count rate $\lambda_{b}$ with units of gamma-ray emissions recorded per unit time. We assume that the background spectrum is known. Let $\boldsymbol{\theta}$ be the vector of all parameters, where $\boldsymbol{\theta}$ lies in the $d$-dimensional parameter space $\Theta$. In what follows, we assume that $\boldsymbol{\theta}$ takes the form:

$$
\boldsymbol{\theta}=\left(\alpha, \boldsymbol{\phi}, e, \lambda_{b}\right)
$$

for which $d=4$. Throughout this work, we assume that the source intensity is unknown, the source position in space $\phi$ and energy $e$ is known, and the background intensity $\lambda_{b}$ can be either known or unknown. Let the modeled sensitivity $\tilde{s}(\phi, e)$ approximate the probability that a photon emitted from a source positioned at $\phi$ is recorded. We model the total rate of recorded photons by the sum of the rates of recorded source and background photons

$$
\tilde{\lambda}(\boldsymbol{\theta}) \triangleq \lambda_{b}+\alpha \tilde{s}(\boldsymbol{\phi}, e) .
$$

2) System Model: We use the model given in [5] to describe the system used in this work. Let $\boldsymbol{r}$ be a vector of recorded attributes associated with a single photon interaction. The recorded attributes contain any and all information that a detector records during a photon interaction. In a position-sensitive Compton detector, the attribute vector $\boldsymbol{r}$ contains the interaction positions and deposited energies for a single interacting photon. In fixed-time mode, the number of recorded photons $J$ is reasonably modeled as a random variable, where $J \sim \operatorname{Poisson}(\tilde{J}(\boldsymbol{\theta}))$. The mean number of recorded photons $\tilde{J}(\boldsymbol{\theta})$ is given by

$$
\tilde{J}(\boldsymbol{\theta}) \triangleq \tilde{\lambda}(\boldsymbol{\theta}) \tau,
$$

where $\tau$ is the scan time. We define the scan time to be the interval during which the system is recording events. Let $\tilde{\boldsymbol{r}}=\left[\boldsymbol{r}_{1}, \boldsymbol{r}_{2}, \ldots, \boldsymbol{r}_{J}\right]$ be a list of the recorded attributes for all interacting photons during a fixed-time scan. By the statistics of list-mode data [9], a reasonable statistical model for the list of recorded attributes $\tilde{\boldsymbol{r}}$ is

$$
\tilde{\mathbf{p}}(\tilde{\boldsymbol{r}} ; \boldsymbol{\theta}) \triangleq e^{-\tau \tilde{\lambda}(\boldsymbol{\theta})}[\tau \tilde{\lambda}(\boldsymbol{\theta})]^{J} / J ! \prod_{j=1}^{J} \tilde{\mathbf{p}}\left(\boldsymbol{r}_{j} ; \boldsymbol{\theta}\right) .
$$

This model is the product of a Poisson term involving the number of recorded counts and the densities of the recorded attributes from each photon. We model the probability density of the individual recorded attributes $\tilde{p}(\boldsymbol{r} ; \boldsymbol{\theta})$ using the approximate model in [1] because it results in reasonable detection performance and does not require numerical integration.

Let $\tilde{\mathrm{p}}_{\mathrm{S}}(\boldsymbol{r} ; \boldsymbol{\phi}, e)$ denote the modeled density of a recorded attribute vector $r$ given that the interacting photon emanated from a source at position $\phi$, and let $\tilde{p}_{\mathrm{B}}(\boldsymbol{r})$ denote the modeled density of a recorded attribute vector $r$ given that the interacting photon originated from the background. Note that $\tilde{p}_{\mathrm{S}}(\boldsymbol{r} ; \boldsymbol{\phi}, e)$ depends on the source position and energy, and $\tilde{p}_{\mathrm{B}}(\boldsymbol{r})$ does not depend on any of the parameters in (1).

We model the distribution of a single recorded attribute as a mixture of $\tilde{p}_{\mathrm{S}}(\boldsymbol{r} ; \boldsymbol{\phi}, e)$ and $\tilde{\mathrm{p}}_{\mathrm{B}}(\boldsymbol{r})$ given by

$$
\tilde{\mathrm{p}}(\boldsymbol{r} ; \boldsymbol{\theta})=\frac{\alpha \tilde{s}(\boldsymbol{\phi}, e) \tilde{\mathrm{p}}_{\mathrm{S}}(\boldsymbol{r} ; \boldsymbol{\phi}, e)+\lambda_{b} \tilde{\mathrm{p}}_{\mathrm{B}}(\boldsymbol{r})}{\alpha \tilde{s}(\boldsymbol{\phi}, e)+\lambda_{b}} .
$$

We substitute (4) into (3) to compute the likelihood of a list of observations. 


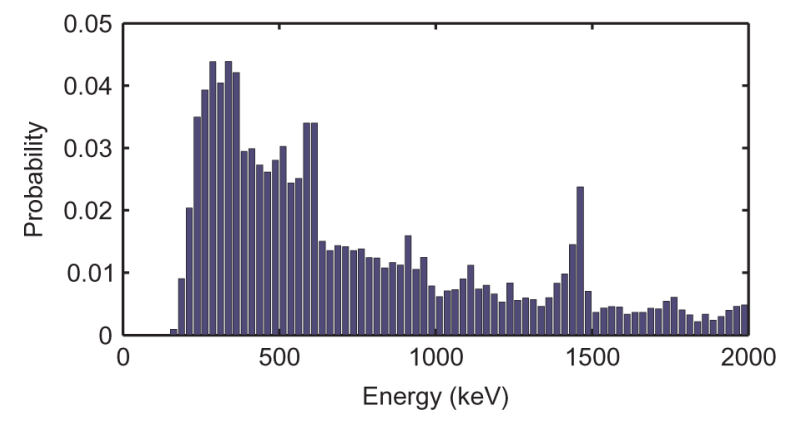

Fig. 1. Estimated probability density function for the incident energy of recorded background photons. This density was estimated using 10000 recorded events from the natural background.

3) Sensitivity Model: The detector sensitivity is the probability that an emitted photon is recorded, which is a function of the photon energy $e$, detector geometry, and the source position $\phi$. Let $\tilde{s}(\phi, e)$ be the modeled system sensitivity. We computed the sensitivity model $\tilde{s}(\phi, e)$ by simulating the detector system in a uniform background using Geant4 [10]. We used simple back projection [11] to compute the sensitivity as a function of position and energy. We normalized the sensitivity so the sensitivity to the source at its true position and energy is unity. This method of computing the sensitivity is approximate and it too may be a source of model mismatch.

4) Background Distribution: To model the background spectrum, we discretize the background energy spectrum into 80 uniformly spaced bins from $0 \mathrm{keV}$ to $2000 \mathrm{keV}$. We assume that the shape of the background spectrum is known, and perform detection with and without the assumption that its intensity is known. When the background intensity is known, we use a previously measured value for the intensity, which assumes that the background is governed by a stationary process. When the background intensity is not known, we estimate it along with the other unknown parameters, such as source intensity. This study examines the effect of different background models on detection performance.

We measured the natural background using 10000 recorded background photons with two or more interactions. We used an expectation maximization (EM) algorithm [12] to reconstruct the incoming spectrum as a function of energy. This estimated incident spectrum serves as a model for the true incident background. We chose to use 10000 recorded events because we were able to obtain a reasonable estimate at a reasonable computational cost.

Let $\mathrm{p}_{\mathrm{B}}(\boldsymbol{\phi}, e)$ be the probability of the incoming position $\phi$ and energy $e$ of a background photon. The source detection model assumes that the background emission density is uniform in space, but varying in energy, i.e., $\mathrm{p}_{\mathrm{B}}(\boldsymbol{\phi}, e)$ is only a function of energy $e$. Let $f(\phi, e)$ be the measured intensity of the background. We have that [9]

$$
\mathrm{p}_{\mathrm{B}}(\boldsymbol{\phi}, e)=\frac{f(\boldsymbol{\phi}, e) \tilde{s}(\boldsymbol{\phi}, e)}{\int_{\Phi \times \mathcal{E}} f(\boldsymbol{\phi}, e) \tilde{s}(\boldsymbol{\phi}, e) d \boldsymbol{\phi} d e} .
$$

We evaluate (5) numerically to compute $\mathrm{p}_{\mathrm{B}}(\phi, e)$. Fig. 1 shows the modeled probability density of recorded energy given that the photon originated from background $\mathrm{p}_{\mathrm{B}}(\boldsymbol{\phi}, e)$ computed using (5).

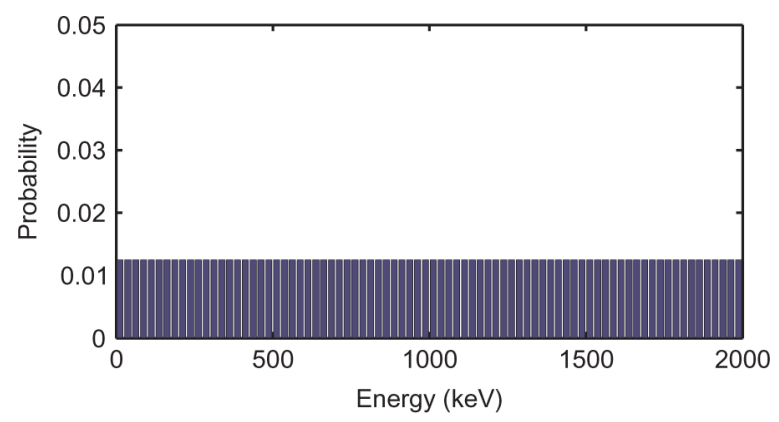

Fig. 2. Uniform incident background energy model.

Fig. 2 shows the modeled probability of the recorded energy given that it originated from the background under the assumption that the recorded energies are uniform. Use of this model will introduce model mismatch.

We evaluate the distribution of recorded attributes given that they originated from background $\tilde{p}_{B}(\boldsymbol{r})$ by

$$
\tilde{\mathrm{p}}_{\mathrm{B}}(\boldsymbol{r})=\sum_{i=1}^{N_{\boldsymbol{\phi}}} \sum_{j=1}^{N_{\text {energy }}} \tilde{\mathrm{p}}_{\mathrm{S}}\left(\boldsymbol{r} ; \boldsymbol{\phi}_{i}, e_{j}\right) \mathrm{p}_{\mathrm{B}}\left(\boldsymbol{\phi}_{i}, e_{j}\right),
$$

where $N_{\boldsymbol{\phi}}$ is the number of position bins and $N_{\text {energy }}$ is the number of energy bins, and $\tilde{\mathrm{p}}_{\mathrm{S}}\left(\boldsymbol{r} ; \boldsymbol{\phi}_{i}, e_{j}\right)$ is the density of recorded attributes $\boldsymbol{r}$ for source position $\boldsymbol{\phi}_{i}$ and energy $e_{j}$. This is a weighted average of the detector response to sources at each position and energy bin.

\section{B. Detection Methods}

We analyze the performance of the source intensity test (SIT) [13] applied to the gamma-ray source detection problem. The SIT is based on the quasi maximum-likelihood (QML) estimate for the source intensity. A QML estimator is equivalent to the ML estimator if the true distribution is in the class of model distributions. The QML estimate for the parameter vector $\boldsymbol{\theta}$ is defined as [14]

$$
\tilde{\boldsymbol{\theta}}_{\tau} \triangleq \arg \max _{\boldsymbol{\theta} \in \Theta} \log \tilde{\boldsymbol{p}}(\tilde{\boldsymbol{r}} ; \boldsymbol{\theta}) .
$$

We use the SIT because experiments showed that its performance was superior to the generalized likelihood ratio test (GLRT) when applied to simple systems [13].

In the absence of model mismatch, the parameter estimate vector $\tilde{\boldsymbol{\theta}}_{\tau}$ converges in probability to the true parameter values as the scan time goes to infinity. However, when model mismatch is present, the estimates may converge to some other value. For example, when there is no source present, the true source intensity is zero, but model mismatch may cause the source intensity estimate to converge to some nonzero value. To precisely define the value to which the parameter estimates converge, we first define the expected log-likelihood by

$$
\begin{aligned}
\tilde{g}(\boldsymbol{\theta}) & \triangleq \mathrm{E}[\log \tilde{\mathbf{p}}(\tilde{\boldsymbol{r}} ; \boldsymbol{\theta})] \\
& =\int_{\mathcal{R}} \log \tilde{\mathbf{p}}(\tilde{\boldsymbol{r}} ; \boldsymbol{\theta}) \mathrm{p}(\tilde{\boldsymbol{r}}) d \tilde{\boldsymbol{r}},
\end{aligned}
$$

where the expectation is with respect to the true distribution and $\mathcal{R}$ is the set of all lists of recorded attributes. This definition uses 
the expected value of the log-likelihood because it is the likelihood function one would obtain with an infinitely large number of observations, and thus it defines the asymptotic behavior of the estimates. The parameter estimates converge to the asymptotic mean, which is defined as

$$
\tilde{\boldsymbol{\mu}} \triangleq \arg \max _{\boldsymbol{\theta} \in \Theta} \tilde{g}(\boldsymbol{\theta}) .
$$

The asymptotic mean is the maximizer of the log-likelihood resulting from an infinite number of measurements, and thus estimates will become very close to the asymptotic mean as the number of observations becomes large. The asymptotic mean is an important component of the performance prediction method.

Let $\alpha^{\mathrm{t}}$ be the true source intensity, which is unknown in practice. The objective of the SIT is to determine whether a source is present $\left(H_{1}\right)$ or a source is absent $\left(H_{0}\right)$, i.e.,

$$
\begin{array}{ll}
H_{1}: & \alpha^{\mathrm{t}}>0 \\
H_{0}: & \alpha^{\mathrm{t}}=0 .
\end{array}
$$

The source intensity test (SIT) [13] for detecting the presence of a radiation source of unknown intensity $\alpha$ is given by

$$
\tilde{\alpha}_{\tau} \underset{H_{0}}{\stackrel{H_{1}}{\gtrless}} \gamma,
$$

where $\tilde{\alpha}_{\tau}$ is the QMLE for $\alpha$, which is the first element of $\tilde{\boldsymbol{\theta}}_{\tau}$, and $\gamma$ is a threshold chosen by the user to obtain the desired false alarm rate. The user decides that a source is present, or $H_{1}$ is true, when the source intensity estimate $\tilde{\alpha}_{\tau}$ is greater than the threshold $\gamma$. The distribution of $\tilde{\alpha}_{\tau}$ determines the threshold value that satisfies the desired false alarm rate, but the distribution of $\tilde{\alpha}_{\tau}$ is intractable in the gamma-ray imaging problem. Therefore, we use asymptotics to predict the distribution of $\tilde{\alpha}_{\tau}$ in a computationally efficient manner.

\section{Performance Measure}

We state our results in terms of the probability of detection as a function of scan time, which is the value of the ROC at a particular false alarm rate as a function of time. This is in contrast to previous works that state performance prediction [3], [5], [13] in terms of ROC and the area under the ROC curve (AUC). We choose to fix a false alarm probability and examine how the probability of detection varies as a function of scan time because the probability of detection is arguably more important to the practitioner than the AUC.

\section{Performance Prediction}

We predict the ROC by approximating the distribution of $\tilde{\alpha}_{\tau}$, justified by Theorems 1 and 2 of [5], by

$$
\tilde{\alpha}_{\tau} \stackrel{\operatorname{approx}}{\sim} \mathcal{N}\left(\tilde{\mu}_{[1]}, \frac{1}{\tau} \Sigma(\tilde{\boldsymbol{\mu}})_{[1,1]}\right),
$$

where $\tilde{\mu}_{[1]}$ is the first element of $\tilde{\boldsymbol{\mu}}$, which corresponds to the asymptotic mean of the source intensity. This approximation becomes exact as the scan time goes to infinity. The covariance is

$$
\Sigma(\boldsymbol{\theta}) \triangleq \tilde{\mathrm{H}}^{-1}(\boldsymbol{\theta}) \tilde{\mathrm{G}}(\boldsymbol{\theta}) \tilde{\mathrm{H}}^{-1}(\boldsymbol{\theta}),
$$

where [5]

$$
\begin{aligned}
\tilde{\mathrm{G}}(\boldsymbol{\theta}) \triangleq & \lambda_{\mathrm{s}} \mathrm{E}\left[\left(\nabla_{\boldsymbol{\theta}} \log \tilde{\mathrm{p}}(\boldsymbol{r} ; \boldsymbol{\theta})+\nabla_{\boldsymbol{\theta}} \log \tilde{\lambda}(\boldsymbol{\theta})\right)\right. \\
& \left.\times\left(\nabla_{\boldsymbol{\theta}} \log \tilde{\mathbf{p}}(\boldsymbol{r} ; \boldsymbol{\theta})+\nabla_{\boldsymbol{\theta}} \log \tilde{\lambda}(\boldsymbol{\theta})\right)^{T}\right], \\
& \quad \text { and } \\
\tilde{\mathrm{H}}(\boldsymbol{\theta}) \triangleq & -\lambda_{\mathrm{S}} \nabla_{\boldsymbol{\theta}}^{2} \log \tilde{\lambda}(\boldsymbol{\theta})+\nabla_{\boldsymbol{\theta}}^{2} \tilde{\lambda}(\boldsymbol{\theta})-\lambda_{\mathrm{S}} \mathrm{E}\left[\nabla_{\boldsymbol{\theta}}^{2} \log \tilde{\mathbf{p}}(\boldsymbol{r} ; \boldsymbol{\theta})\right],
\end{aligned}
$$

$\nabla_{\boldsymbol{\theta}}$ is the column gradient with respect to $\boldsymbol{\theta}, \nabla_{\boldsymbol{\theta}}{ }^{2}$ is the Hessian with respect to $\theta, \lambda_{\mathrm{s}}$ is the true recorded count rate, and expectations are with respect to the true distribution. The approximation in (10) gives a distribution that one can use to approximate the distribution of the gamma-ray source intensity estimate. The mean and covariance of this distribution are given by (8) and (12), respectively.

If the source position $\phi$ and background intensity $\lambda_{b}$ are known, then $\Sigma(\boldsymbol{\theta}), \tilde{G}(\boldsymbol{\theta})$, and $\tilde{\mathrm{H}}(\boldsymbol{\theta})$ are scalar, otherwise they are matrices. $\tilde{\mathrm{G}}(\boldsymbol{\theta})$ and $\tilde{\mathrm{H}}(\boldsymbol{\theta})$ characterize the uncertainty in estimates of the parameter $\boldsymbol{\theta}$. Larger values of $\tilde{\mathrm{G}}(\boldsymbol{\theta})$ and $\tilde{\mathrm{H}}(\boldsymbol{\theta})$ correspond to lower uncertainty in the measurements. Since the true distribution is unknown, we describe a method for evaluating $\tilde{\mu}_{[1]}$ and $\Sigma(\tilde{\boldsymbol{\mu}})$ in the next section.

\section{E. Procedure for Computing Predicted Performance}

We used the following procedure to apply the asymptotic approximation in (10) for detection performance prediction.

1) Obtain $N$ recorded events with a source placed $d$ meters from the detector at an azimuth angle $\phi_{s}$ and polar angle $\theta_{s}$ in a coordinate system centered at the detector, and $\left(\phi_{s}=0^{\circ}, \theta_{s}=90^{\circ}\right)$ is the vector pointing from the front of the detector. One should choose $N$ to be large enough to achieve the desired confidence in estimates of source and background intensity.

2) Evaluate the asymptotic mean $\tilde{\mu}_{[1]}$ by solving (6) using the recorded events.

3) Evaluate the asymptotic covariance $\Sigma(\tilde{\mu})$ using (11), (12), and (13). Use Monte Carlo integration with the recorded data to evaluate the expectations, e.g., for i.i.d. random variables $X_{i}, i=1,2, \ldots, N$ with density functions $p(x)$ and a function $f(x)$,

$$
\mathrm{E}[f(x)] \approx \frac{1}{N} \sum_{i=1}^{N} f\left(X_{i}\right) .
$$

4) Compute the true count rate $\lambda_{s}$ by averaging the count over a sufficiently long time to achieve the desired confidence in the estimate. We chose two hours.

5) Obtain $\mathrm{N}$ recorded events with the detector in the same position as step 1 but without a source present.

6) Repeat steps 2-4 in the absence of a source. 
7) Use the computed asymptotic means and covariances to compute the approximate distribution of $\tilde{\alpha}_{\tau}$ with and without a source present.

8) Use the approximate distributions to compute the probabilities of false alarm and detection. Compute the probability of false alarm by evaluating the probability that the source intensity estimate $\tilde{\alpha}_{\tau}$ is greater than the threshold $\gamma$ in (9) using the Gaussian cumulative distribution function and the mean and covariance in the absence of a source computed in step 7. Compute the probability of false alarm by evaluating the probability that the source intensity estimate $\tilde{\alpha}_{\tau}$ is greater than the threshold $\gamma$ in (9) using the Gaussian cumulative distribution function and the mean and covariance in the presence of a source computed in step 7. Use several different thresholds $\gamma$ to produce an ROC curve.

9) Generate multiple ROC curves to generate the probability of detection as a function of scan time for a fixed false alarm rate.

\section{F. Source Intensity Variation}

We recorded data with and without a source present to predict detection performance. We achieved the desired source-to-background ratio by combining events from the measurements with and without a source. For example, the measurements of a $1-\mu \mathrm{Ci}$ ${ }^{137} \mathrm{Cs}$ source placed 1.83 meters from the detector contain approximately $48 \%$ source events and $52 \%$ background events across the entire energy spectrum. We observed the recorded background count rate $\lambda_{b}$ to be approximately 9114 counts per minute using a measurement of 90.7 hours. The observed count rate of the measurement containing both source and background events is 18984 counts per minute, which we obtained with a scan time of 121 minutes. We combined events from the lists obtained with and without a source to explore a range of source-tobackground ratios.

\section{G. Conventional Method for Empirical Calculations}

We compared the predicted performance to the empirical performance in terms of probability of detection. This method is similar to that used in [3], [5], and [13]. We used the following procedure to compute the empirical performance:

1) Repeat $N_{e}$ times with a source present and $N_{e}$ times without a source present:

a) Draw the number of recorded source counts from a Poisson distribution with mean $\tau \alpha^{\mathrm{t}} s^{\mathrm{t}}$, where $\alpha^{\mathrm{t}}$ is the true source intensity, $s^{\mathrm{t}}$ is the true sensitivity, and $\tau \alpha^{\mathrm{t}} s^{\mathrm{t}}$ is the mean number of received counts from the source.

b) Draw the number of recorded background counts from a Poisson distribution with mean equal to the mean number of recorded background counts $\tau \lambda_{b}$.

c) Generate a list of events that contains $\tau \alpha^{\mathrm{t}} s^{\mathrm{t}}$ source counts and $\tau \lambda_{b}$ background counts using the events recorded in the presence and absence of a source. Combine the events from the measurements with and without a source to achieve the proper mean number of source counts.

d) Solve (6) using the list generated in the previous step.
2) Use the empirical source intensity estimates obtained in step 1 to compute the empirical probabilities of detection and false alarm.

3) Generate multiple ROC curves to generate the probability of detection as a function of scan time for a fixed false alarm rate.

We computed the empirical performance by emulating $N_{e}=100$ scans with a source present and $N_{e}=100$ scans without a source present.

This empirical calculation method requires the emulation of 200 scans for each point on the graph of probability of detection versus scan time. In contrast, the asymptotic prediction method based on (10) requires one computation with approximately 20000 recorded events for all scan times because the asymptotic mean is invariant to scan time and the asymptotic covariance in (11) scales as the inverse of the scan time. Thus, computing detection performance as a function of scan time with the asymptotic prediction method requires less computation than computing the performance empirically. The performance gain is proportional to the number of scan time samples.

\section{RESULTS}

We computed the probability of detection as a function of scan time when the source position, source energy, and background spectrum are assumed known. We examined the case of a known and unknown background intensity and investigated how inaccuracies in the background spectral model affect detection performance. We found that the performance predicted using (10) agreed well with the empirical performance in the known background case. In the unknown background case, the agreement between the theoretical and empirical predictions is not as good as in the known background case. In both cases, there is model mismatch due to the noisy background spectrum estimate, the incorrect assumption that the background is spatially uniform, and approximations in the detector system response [1].

The results were generated with data recorded with an 18-detector CdZnTe array. The detector records photon interactions with up to four interaction events, including single-interaction events.

\section{A. ${ }^{137}$ Cs With Measured Background Spectrum and Known Background Intensity}

We first examined the problem of detecting a ${ }^{137} \mathrm{Cs}$ source when the background spectrum is modeled by the measured background spectrum in Fig. 1, and the background intensity is assumed known. Fig. 3 shows the probability of detection as a function of scan time for probability of false alarms of 5\% and $10 \%$ with source intensities of 7.6 counts per second and 15.2 counts per second. The background intensity is 152 counts per second. We use large false alarm rates so the system can achieve a probability of detection near one in less than ten seconds. One could apply this theory to obtain predicted performance for lower false alarm rates. The markers on the plot are intended to aid the reader in distinguishing the curves. These markers do not represent data points.

The agreement between the empirical and predicted probability of detection is better with the higher source intensity for 


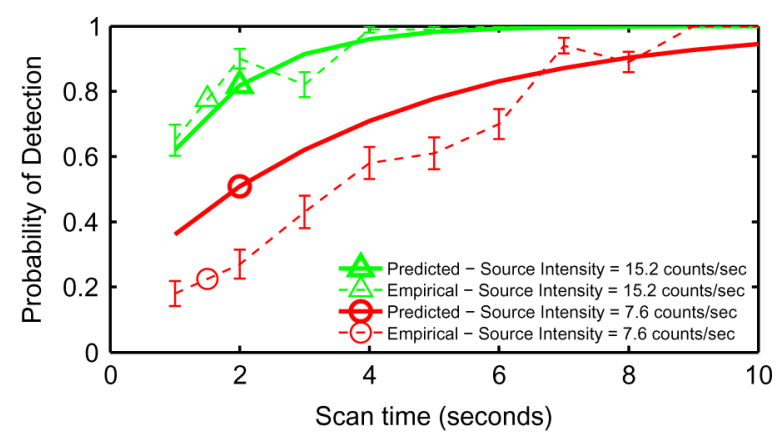

(a)

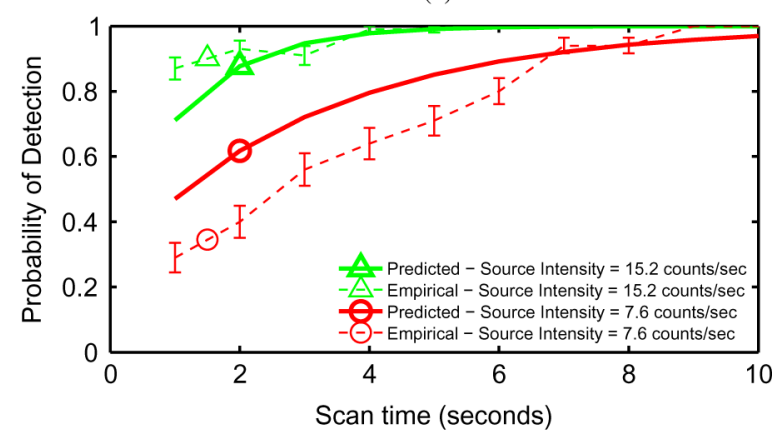

(b)

Fig. 3. Probability of detection versus scan time for detecting a ${ }^{137} \mathrm{Cs}$ source in a natural background with intensity 152 counts per second using an $18 \mathrm{de}-$ tector CdZnTe array. The background shape and intensity are assumed known and the background shape is modeled using a prior spectral measurement. (a) Probability of false alarm: 5\%, (b) Probability of false alarm: $10 \%$.

both false alarm rates. This is likely due to the fact that the Gaussian approximation for the distribution of the source intensity estimate (10) improves as the number of recorded counts increases [5]. This shows that the ROC approximation based on asymptotics is more accurate for larger numbers of recorded counts in this case.

Fig. 4 shows the probability of detection versus scan time for source intensities of 7.6 and 15.2 counts per second with a false alarm rate of $10 \%$. The curves in each graph correspond to the performance achieved when using all imaging and spectral information, spectral information only, and the number of recorded counts only. The detector used in this experiment records positions and energies of all interacting photons, and the performance using all imaging information takes all of this information into account. We obtained the performance using spectral information by only parameterizing the source energy and not its position. We eliminated the parameterization with respect to position by numerically integrating the likelihood with respect to position over the sphere surrounding the detector. The spectral information case essentially disregards the information from the recorded positions in the detector. We computed the performance using counting only by considering the Gaussian approximation to the distributions of the source intensity estimates obtained using only the number of recorded counts. The counting case essentially disregards all information except the number of recorded counts.

Fig. 4 shows that using imaging information results in the best detection performance with both source intensities. The increase in probability of detection over the spectral and counting

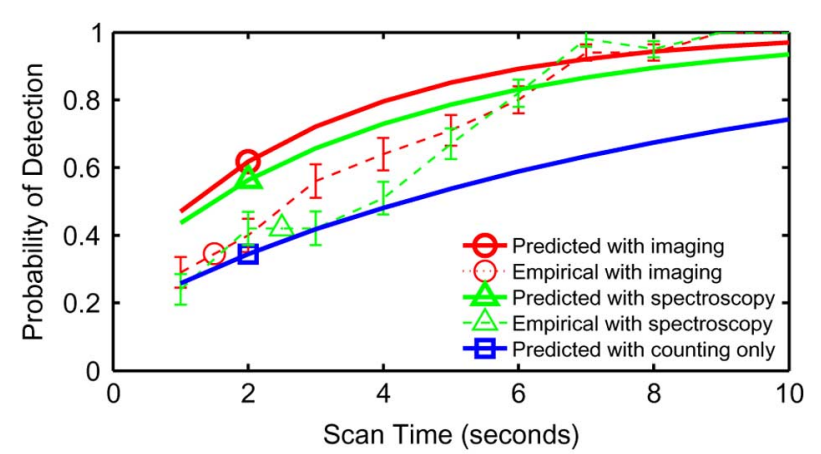

(a)

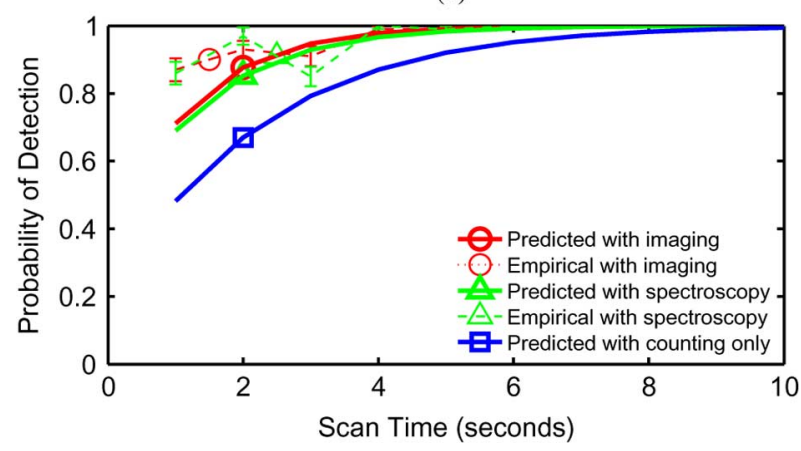

(b)

Fig. 4. Probability of detection versus scan time for detecting a ${ }^{137} \mathrm{Cs}$ source in a natural background with intensity 152 counts per second at a false alarm rate of $10 \%$ using an 18 detector CdZnTe array with imaging and spectral information, spectral information only, and counting information only. The background shape and intensity are assumed known and the background shape is modeled using a prior spectral measurement. (a) Source intensity: 7.6 counts/sec, (b) Source intensity: 15.2 counts/sec.

cases is more in the case where the source intensity is lower. For both source intensities, the performance difference between the case of spectral information and imaging information is small. The estimation problem is much simpler when only spectral information is used. In this experiment, there are 324 position bins and 80 energy bins. There are 80 parameter bins when energy is used without position, and $80 \times 324$ parameter bins when energy and imaging are used. Thus, parameterizing the image space requires 324 times more computation. There may be practical applications where the slight decrease in performance is justified by the reduced computation when the source position and energy are known.

As in Fig. 3, the agreement between the predicted and empirical performance is better for the higher source intensity for small scan times. As scan time increases, the empirical and predicted performance agree well.

\section{B. ${ }^{137}$ Cs With Measured Background Spectrum and Unknown Background Intensity}

We also examined the problem of detecting a ${ }^{137} \mathrm{Cs}$ source in background when the background spectrum is modeled by the measured background spectrum in Fig. 1, but the background intensity is unknown and estimated. Fig. 5 shows the probability of detection as a function of scan time with a background intensity of 152 counts per second. The predicted and empirical performance values are lower than in the known background case 


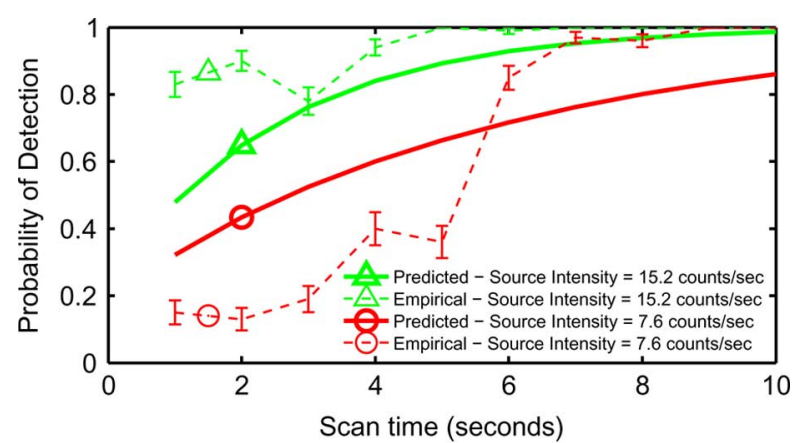

(a)

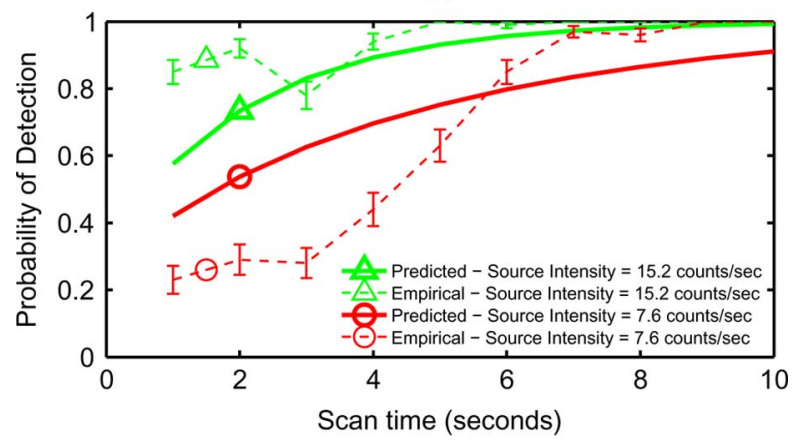

(b)

Fig. 5. Probability of detection versus scan time for detecting a ${ }^{137} \mathrm{Cs}$ source in a natural background with intensity 152 counts per second using an 18 detector $\mathrm{CdZnTe}$ array. The background shape is assumed known and is spectral measurement, but the background intensity is assumed unknown. (a) Probability of false alarm: 5\%, (b) Probability of false alarm: $10 \%$.

of Fig. 3, which is expected since we introduced a nuisance parameter.

The agreement between the predicted and empirical probability of detection is poor in the unknown background case considered here. Fig. 6 shows histograms of the empirical source intensity estimates with and without a source present when the scan time $\tau=2$ seconds and the source intensity is 15.2 counts per second. The empirical performance is based on the empirical histogram and the predicted performance is based on the predicted distribution. The histograms in Fig. 6 do not agree well with the predicted distributions because the scan time is too small for the predicted distribution to be accurate. The accuracy of the predicted distribution increases as scan time increases because it is based on an asymptotic approximation [5].

Fig. 7 shows empirical histograms and predicted distributions when the scan time $\tau=10$ seconds. The distribution agrees better under $H_{1}$ than it did with scan time $\tau=2$ seconds, but the histogram under $H_{1}$ shows that the empirical distribution has a lower variance than predicted, causing the empirical performance to be better than the predicted performance. The poor agreement between the empirical and predicted distributions is likely because the predicted distribution is less accurate for small scan times when the background intensity is unknown. Our results only use 100 scans with and without a source, and one may obtain better agreement by using a larger number of trial scans.

Fig. 8 shows the probability of detection as a function of scan time for a probability of false alarm of $10 \%$ with position, spectral, and counting information. In the unknown back-

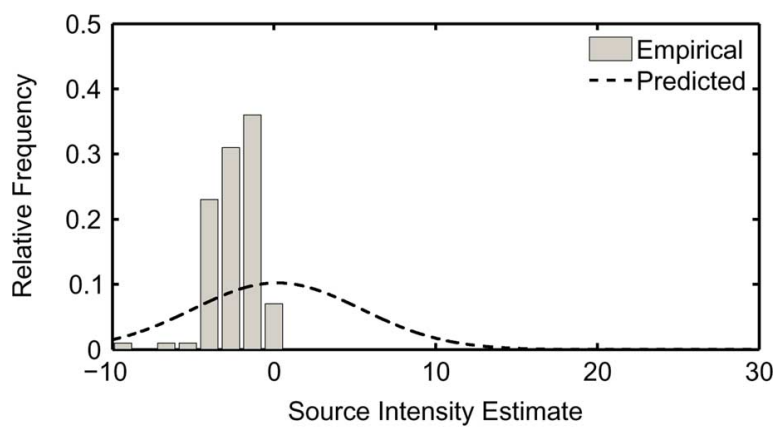

(a)

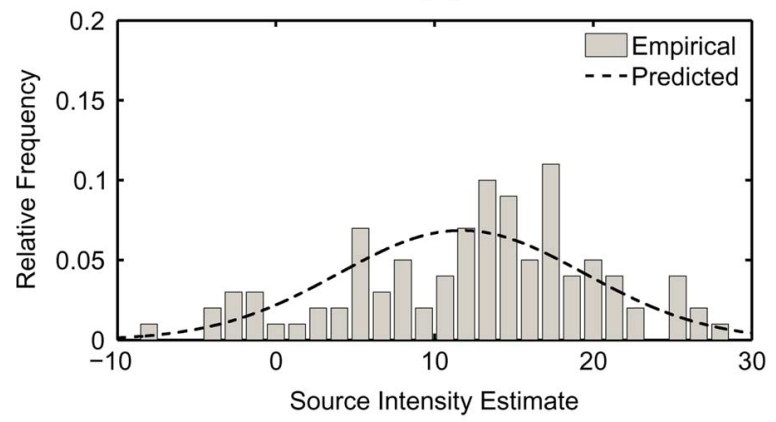

(b)

Fig. 6. Histograms of source intensity estimates and predicted distributions for scan time $\tau=2$ seconds and source intensity $\alpha^{\mathrm{t}}=15.2$. (a) Source Absent $\left(H_{0}\right)$, (b) Source Present $\left(H_{1}\right)$.

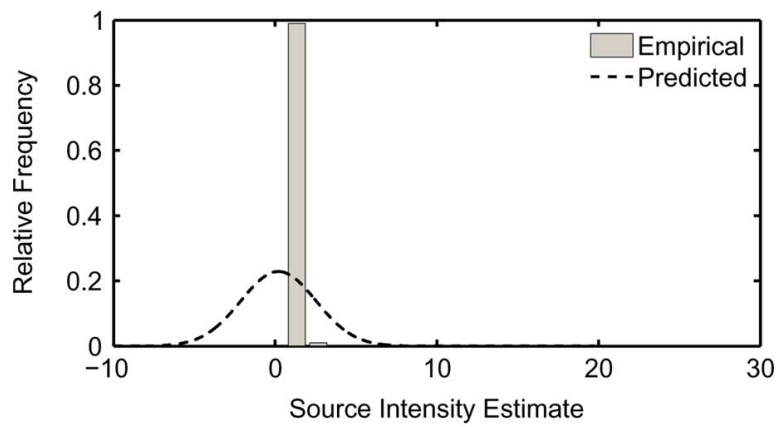

(a)

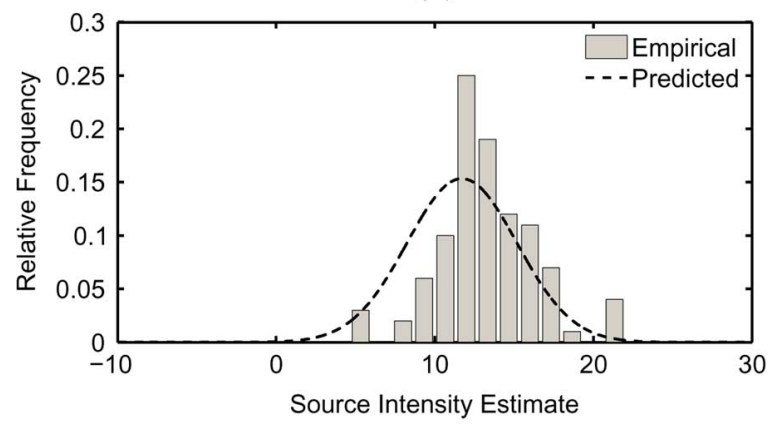

(b)

Fig. 7. Histograms of source intensity estimates and predicted distributions for scan time $\tau=10$ seconds and source intensity $\alpha^{\mathrm{t}}=15.2$. (a) Source Absent $\left(H_{0}\right)$, (b) Source Present $\left(H_{1}\right)$.

ground case, the predicted performance using counting statistics assumes a known background intensity because the source and background intensities cannot be estimated otherwise. In this case, the performance using imaging and spectral information in an unknown background is better than the performance of the 


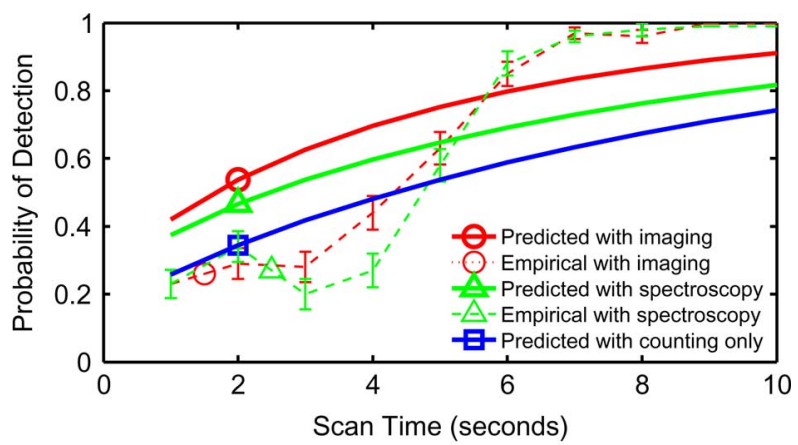

(a)

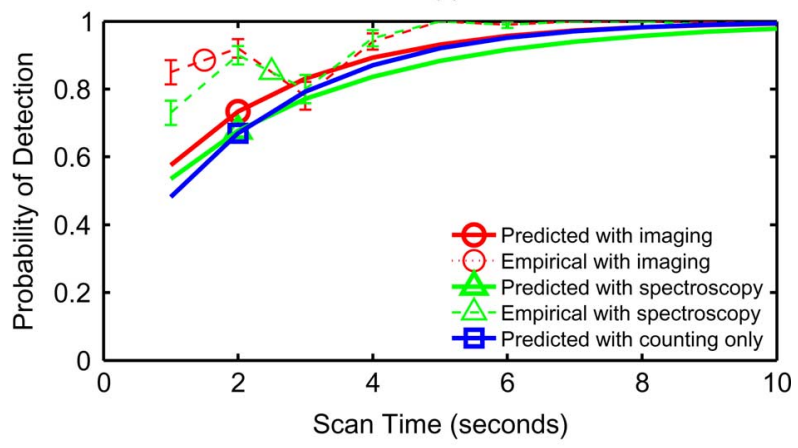

(b)

Fig. 8. Probability of detection versus scan time for detecting a ${ }^{137} \mathrm{Cs}$ source in a natural background with intensity 152 counts per second at a false alarm rate of $10 \%$ using an 18 detector CdZnTe array with imaging and spectral information, spectral information only, and counting information only. The background shape is assumed known and is modeled using a prior spectral measurement, but the background intensity is assumed unknown. (a) Source intensity: 7.6 counts/sec, (b) Source intensity: 15.2 counts/sec.

counting method, even though the counting method assumes a known background.

The difference in performance between the imaging, spectroscopy, and counting cases is more pronounced with the weaker source in Fig. 8(a). Even when the source intensity is 15.2 counts per second in Fig. 8(b), the performance with imaging information in an unknown background is still better than the performance using counting statistics only in a known background, although the results are not significantly different.

\section{Effect of Incorrect Modeled Background Spectrum}

We also explored the robustness of the SIT to changes in the modeled background spectrum. The background spectrum may change over time or if the detector is moved to a new location, making it difficult to acquire a background spectrum estimate that will be accurate at all times. Fig. 9 shows the probability of detection as a function of scan time when the background spectrum is modeled using the estimated spectrum in Fig. 1 and the uniform spectrum in Fig. 2 when the background intensity is known.

Fig. 9 shows that in this case, the predicted performance does not change significantly when the background spectral model is changed. This is likely because the source energy is known to be $662 \mathrm{keV}$.

Fig. 10 shows the probability of detection as a function of scan time for the estimated and uniform background models with an unknown background intensity. The difference between the predicted performance curves is also negligible in this case.

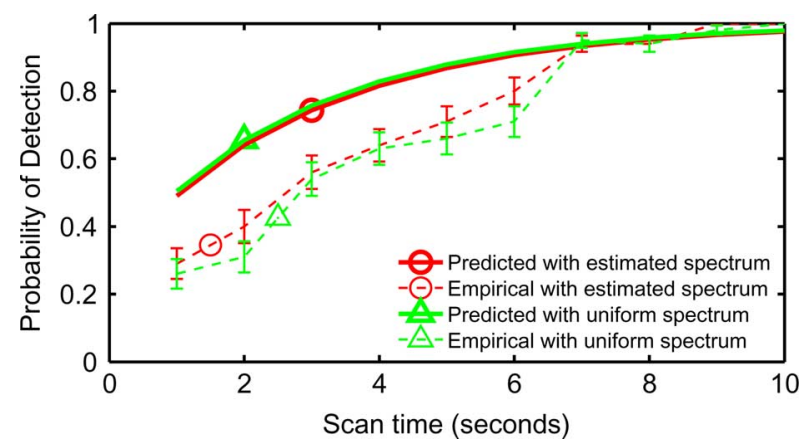

Fig. 9. Probability of detection vs. scan time with an estimated background spectral model and a uniform background spectral model. The source intensity is 7.6 counts per second. The background intensity is 152 counts per second and is assumed known. The probability of false alarm is $10 \%$.

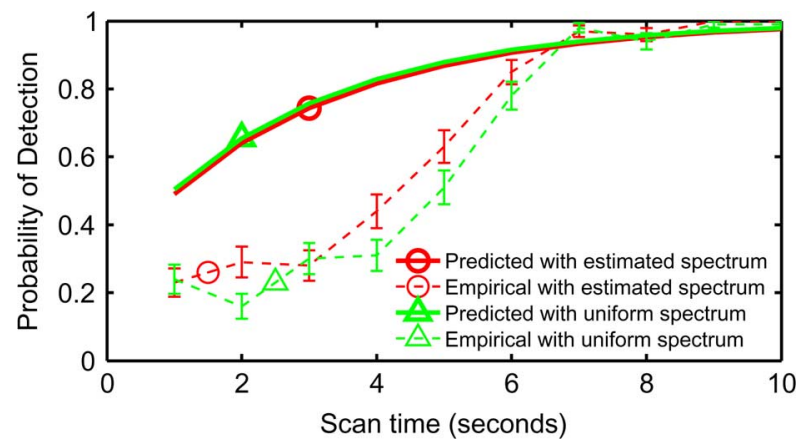

Fig. 10. Probability of detection vs. scan time with an estimated background spectral model and a uniform background spectral model. The source intensity is 7.6 counts per second. The background intensity is 152 counts per second and is not assumed known. The probability of false alarm is $10 \%$.

However, the predicted performance is a poor approximation of the empirical performance.

The comparison between the estimated and uniform spectral models shows that the SIT is robust to the background spectral model in this case when the source position and isotope are known. This robustness is a result of the source having a single energy and the background having a continuous and diffuse energy spectrum.

\section{Practical Applications}

The results in this section show that the proposed ROC prediction method is accurate in certain scenarios. This method would be useful in system design problems where one needs to choose parameters such as the number of detectors, the physical arrangement of the detectors, or even the type of detectors. The approximate distribution of the source intensity estimate in (10) is a function of the scan time, so one can produce ROC curves for different scan times with the same data set, reducing the time required for measurement, simulation, and computation. The time required to compute the asymptotic approximation of the probability of detection versus scan time with 20000 recorded events is approximately three hours on modern hardware at the time of publication. For example, the empirical calculations in this work were preformed for 10 distinct scan times. The most computationally intensive task in this problem is calculating the likelihoods, which must be done for both the empirical and approximate methods. Thus, it should take approximately 10 times as much computation to produce the empirical 
curves in this section than the approximate curves, and this difference in computation time was observed while generating the results.

\section{CONCLUSION AND FUTURE WORK}

We applied the asymptotic detection performance prediction method developed in [5] to a real system with real recorded data. Our results showed that the asymptotic prediction method accurately predicts detection performance for $5 \%$ and $10 \%$ source-to-background ratios when the background intensity is known. When the background intensity is unknown, the predicted ROC was within the uncertainty of the empirical ROC when the source-to-background ratio was $10 \%$, but not when the source-to-background ratio was $5 \%$.

This work serves as an example application of the theory of [5] to performance characterization of real systems. Our results show that the asymptotic performance prediction method gives reasonably accurate performance predictions that one can use to help determine sensor placement, configuration, or viability.

We considered the case where the source position and energy are known. Future work would investigate the accuracy of the detection performance prediction method of [5] when the source position and energy are unknown. The unknown energy case presents additional challenges because in a typical application, only a set of possible sources are of interest, and each source may emit gamma-rays with multiple energies. The prediction method of [5] only applies to continuous parameters, but an unknown isotope is a discrete parameter. Future work would extend the performance prediction method to the case of a discrete parameter.

We also assumed that the background spectrum and spatial distribution are known. We used this assumption because background shape and spectrum estimation is a high-dimensional problem. Future work would investigate methods of reducing the dimensionality of the background estimation problem so reasonable estimates could be obtained with a reasonable amount of data and computation.

\section{REFERENCES}

[1] D. Xu and Z. He, "Gamma-ray energy-imaging integrated spectral deconvolution," Nucl. Instrum. Meth. Phys. Res. A., vol. 574, no. 1, pp. 98-109, Apr. 2007.

[2] S. M. Kay, Fundamentals of Statistical Signal Processing: Detection Theory. New York: Prentice-Hall, 1998.

[3] C. G. Wahl and Z. He, "Point-source detection using energy and imaging information from 3D-position-sensitive semiconductor detectors," in Proc. IEEE Nucl. Sci. Symp. Conf. Rec. (NSS/MIC), Oct. 2009, pp. 1069-1073.

[4] R. C. Runkle, T. M. Mercier, K. K. Anderson, and D. K. Carlson, "Point source detection and characterization for vehicle radiation portal monitors," IEEE Trans. Nucl. Sci., vol. 52, no. 6, pp. 3020-3025, Dec. 2005.

[5] D. J. Lingenfelter, J. A. Fessler, C. D. Scott, and Z. He, "Asymptotic source detection performance of gamma-ray imaging systems under model mismatch," IEEE Trans. Signal Process., vol. 59, no. 11, pp. 5141-5151, Nov. 2011.

[6] G. F. Knoll, Radiation Detection and Measurement, 3rd ed. New York: Wiley, 2000.

[7] Z. He, W. Li, G. F. Knoll, D. K. Wehe, J. Berry, and C. M. Stahle, "3-D position sensitive CdZnTe gamma-ray spectrometers," Nucl. Instrum. Meth. Phys. Res. A, pp. 173-178, Feb. 1999.

[8] F. Zhang, Z. He, and C. E. Seifert, "A prototype three-dimensional position sensitive CdZnTe detector array," IEEE Trans. Nucl. Sci., vol. 54, no. 4, pp. 843-848, Aug. 2007.

[9] H. H. Barrett, T. White, and L. C. Parra, "List-mode likelihood," J. Opt. Soc. Amer. A, vol. 14, no. 1, pp. 2914-2923, Nov. 1997.

[10] J. Allison et al., "Geant4 developments and applications," IEEE Trans. Nucl. Sci., vol. 53, no. 1, pp. 270-278, Feb. 2006.

[11] D. Xu, "Gamma-ray imaging and polarization measure using 3-D position-sensitive CdZnTe detectors," Ph.D. dissertation, Univ. of Michigan, Ann Arbor, 2006

[12] A. P. Dempster, N. M. Laird, and D. B. Rubin, "Maximum likelihood from incomplete data via the EM algorithm," J. R. Stat. Soc. Ser. B, vol. 39, no. 1, pp. 1-38, 1977.

[13] D. J. Lingenfelter, J. A. Fessler, C. D. Scott, and Z. He, "Benefits of position-sensitive detectors for radioactive source detection," IEEE Trans. Signal Process., vol. 58, no. 9, pp. 4473-4483, Sep. 2010.

[14] H. White, "Maximum likelihood estimation of misspecified models," Econometrica, vol. 50, no. 1, pp. 1-25, 1982. 